\title{
Efeitos de diferentes padrões respiratórios no desempenho e na organização temporal das braçadas do nado "Crawl"
}

CDD. 20.ed. 152.3

797.2

\author{
Marcos Roberto APOLINARIO* \\ Thiago Augusto Costa de OLIVEIRA* \\ Lúcio Fernandes FERREIRA*/** \\ Luciano BASSO* \\ Umberto César CORRÊA* \\ Andrea Michele FREUDENHEIM* \\ *Escola de Educa- \\ ção Física e Esporte, \\ Universidade de São \\ Paulo. \\ **Universidade Nilton \\ Lins de Manaus.
}

\section{Resumo}

0 objetivo deste estudo foi investigar os efeitos de diferentes padrões respiratórios no desempenho e na organização temporal das braçadas do nado "Crawl" de atletas de elite. Vinte e um jovens atletas do gênero masculino participaram de forma voluntária do estudo. Eles foram filmados nadando $25 \mathrm{~m}$ do nado "Crawl", em velocidade máxima, em quatro condições de respiração: inspiração para o lado preferido (LP); para o lado não preferido (LNP); bilateral (B); e, sem respiração (S). Duas filmadoras digitais (60 fps) captaram as imagens que foram analisadas através do "software" Kinovea 0.8.7 nos 10 metros centrais do percurso. Para análise foram consideradas medidas de desempenho e de organização temporal das braçadas. Os resultados permitiram concluir que o padrão respiratório afeta o desempenho de atletas jovens de elite. Mostraram que, em termos de velocidade, tempo, frequência e comprimento de braçada, é mais vantajosa a condição de nadar "Crawl" em apneia (S) que em qualquer das condições com inspiração (B, LP e LNP). Portanto, o padrão preferido de respiração, não necessariamente, corresponde ao mais eficiente em termos do desempenho. Assim, professores e técnicos de Natação deveriam incentivar a prática e o emprego do padrão em apneia como estratégia tática para nadadores velocistas. Em relação à braçada, os resultados mostraram que diante da modificação do padrão respiratório, nadadores peritos jovens mantêm a estrutura temporal das braçadas direita e esquerda (aspectos invariantes da braçada) bem como a coordenação entre os braços inalterada enquanto efetuam ajustes na fase aérea da braçada (aspectos variantes). Assim sendo, atletas jovens de elite apresentam braçada com relativa autonomia frente ao componente respiração e nadam efetuando ajustes considerando a economia de recursos.

UnITERMOS: Natação; Nado "Crawl"; Comportamento motor.

\section{Introdução}

Os estudos que envolvem os nados têm sido desenvolvidos predominantemente com o objetivo de revelar quais são os principais determinantes de sua eficiência. Nesse contexto, tem-se investigado, entre outros, o efeito da respiração na braçada do nado "Crawl" (LeRDA, Cardelli \& Chollet, 2001; Payton, Bartlett, Baltzopoulos \& Coombs, 1999; Seifert, Chollet \& Allard, 2005; Seifert, Chehensse, Chollet, Lemaitre \& Chollet, 2008; Vezos, Gourgoulis, Aggeloussis, Kasimatis, ChrisTOFORIDIS \& MAVROMATIS, 2007). Em conjunto, estes estudos mostraram que os nadadores habilidosos não alteram o $\mathrm{Fb}$ e $\mathrm{Cb}$ em função da condição de respiração. No entanto, o efeito do padrão respiratório na velocidade máxima de nado, aspecto importante para auferir eficiência na Natação, ainda não é conhecido.

$\mathrm{Na}$ maioria dos estudos realizados a tarefa compreendeu nadar em velocidade máxima simulada (Lerda, Cardelli \& Chollet, 2001; Payton et al., 1999; Seifert et al., 2008). No estudo de LERDA, CARDELli e CHOlLet (2001), por exemplo, a solicitação foi nadar a distância de $25 \mathrm{~m}$ simulando velocidade de provas de 100 e de $800 \mathrm{~m}$. Portanto, neste conjunto de estudos, a velocidade, aspecto crítico do 
desempenho do nadador, foi tratada como variável independente. Ainda, em dois destes estudos, o nado "Crawl" foi descaracterizado. No estudo de PAYTON et al. (1999), para possibilitar a análise do ângulo de rolamento do corpo, os nadadores nadaram com uma estrutura de madeira fixada nas costas, e, em SEIFERT et al. (2008), as pernas foram atadas para anular sua ação. Com base no exposto, percebeuse a necessidade de revisitar esta questáo, ou seja, de investigar os efeitos do padrão de respiração no desempenho do nado "Crawl" de atletas. Desta necessidade surge a primeira questão deste estudo: o desempenho de atletas nadando "Crawl" é afetado pelo padrão respiratório - sem inspiração (apneia), com inspiração unilateral (LP e LNP) e bilateral?

O nado "Crawl" é uma das habilidades em que há evidente necessidade de padronização espaçotemporal dos movimentos. O deslocamento eficaz e eficiente do corpo na água exige uma ação coordenada entre braços, pernas e respiração, favorável à sua propulsão. Assim, a organização temporal dos movimentos desses vários componentes é crucial para a ação. Talvez por isso, ao observar indivíduos habilidosos praticando Natação supōe-se que estejam repetindo uma série de movimentos idênticos. No entanto, de acordo com BARTLETT (1932) não se pode desconsiderar que além de consistente, o comportamento motor habilidoso é, ao mesmo tempo, variável. Segundo Sснмidt $(1985,2003)$, mesmo diante de alterações paramétricas da tarefa, algumas características do comportamento são mantidas invariantes, como o "timing" relativo, o sequenciamento e a força relativa. Essa invariância é que garante a consistência do movimento. Mas, ao mesmo tempo, diante de alteraçōes da tarefa, aspectos relacionados a parâmetros como tempo de movimento, força total, e seleção dos músculos podem variar para atender as demandas específicas da tarefa, dando uma configuração única a cada padrão do movimento. Esta possibilidade de variar os parâmetros de movimento ao mesmo tempo em que aspectos de sua estrutura são mantidos, garante a flexibilidade do comportamento. Assim, como nas demais habilidades motoras, no nadar "Crawl" há aspectos do comportamento que tendem a ser mais invariantes que outros (FREUDENHEIM, BASSO, Xavier Filho, Silva, Madureira \& Manoel, 2005).

Apolinário, Silva, Tertuliano, Oliveira, Marques e Freudenheim (2007), investigaram o efeito da respiração na braçada do nado "Crawl", diferenciando os aspectos invariantes dos variantes. Os resultados do estudo indicaram que os nadadores se adaptaram à mudança para o lado não preferido de inspiração, principalmente a partir de ajustes nos aspectos variantes. Entretanto, este estudo exploratório envolveu poucos participantes e, segundo os autores, a tarefa de nadar em velocidade lenta não representou um desafio para os mesmos. Também neste estudo, o IdC, medida que permite a análise do padrão de interação das ações dos braços direito e esquerdo, não foi mensurado.

Nesse sentido, surge a segunda questão: quais os efeitos de diferentes padrōes de respiração na organização temporal - IdC, aspectos invariantes e variantes - da braçada do nado "Crawl"? A escolha da braçada se justifica pelo fato de ser o principal componente propulsivo do nado "Crawl" (DEsCHODT, ARSAC \& Rouard, 1999) e, como especificado mais adiante, por ser constituída de componentes claramente identificáveis.

Em suma, considerando as duas questōes levantadas, o objetivo do presente estudo foi investigar os efeitos de diferentes padrōes respiratórios no desempenho e na organização temporal das braçadas do nado "Crawl". Para tanto, se fez necessário no presente estudo, utilizar a tarefa nadar em velocidade máxima real e poder distinguir os aspectos que expressam o padrão de movimento daqueles que correspondem à parametrização da ação da braçada.

O IdC foi proposto e tem sido utilizado como medida do padrão de interação das ações dos braços direito e esquerdo pois mensura a diferença de tempo entre as ações propulsivas dos dois braços (CHOLLET, Chalies \& Chatard, 2000). Por sua vez, o "timing" relativo corresponde à porcentagem do tempo total de movimento (duração total da braçada) gasto em cada fase. Com a duração total da braçada e as duraçōes de cada fase foi possível calcular o "timing" relativo das fases. O "timing" relativo tem sido considerado um bom indicativo da organização temporal do comportamento motor (aspectos invariantes), pois se mantém relativamente constante mesmo frente a mudanças na ação - como, por exemplo, aumento da velocidade, aumento da amplitude de movimentos e a mudança de grupo muscular utilizado na execução (Freudenheim er al., 2005). No entanto, o "timing" relativo deve ser considerado em conjunto com o tempo absoluto, pois o primeiro é invariante somente frente à maior variabilidade deste último (HEUER, 1991). Por isso, neste estudo, utilizou-se o IdC, o "timing" relativo e o tempo absoluto como indicativos da organização temporal da ação. Como a braçada do nado "Crawl" é composta por componentes claramente diferenciados - fases aérea e aquática - da mesma forma que em 
Freudenheim et al. (2005) - foi possível calcular o "timing" relativo dos componentes bem como o seu tempo absoluto. Ainda, como a fase aquática também pode ser claramente decomposta em entrada e pegada, puxada e empurrada foi possível calcular o IdC.

Vale lembrar que o nado "Crawl" pode ser considerado como um sistema aberto hierárquico (FREUDENHEIM et al., 2005; MAdUREIRA, 2006). Sistema, porque diz respeito a um conjunto de componentes (braçada, pernada e respiração) que ocorrem em interação. Aberto, pois, o nadador deve efetuar constantes ajustes para se adaptar às variações do meio aquático, da tarefa e do organismo. No caso deste último, em função, por exemplo, da fadiga. E, hierárquico, devido a cada componente apresentar ao mesmo tempo propriedades de todo e partes (KoESTLER, 1969). Isso significa que quando se olha somente para a braçada, por exemplo, ela pode ser vista como um todo composto de duas partes (aérea e aquática); mas quando se olha para o "Crawl" propriamente dito, a braçada é uma de suas partes (Oliveira, Apolinário, FREUdENHEIM \& CORRÊA, 2009).

Portanto, o nado Crawl se caracteriza por apresentar interação entre os componentes, ou seja, alta organização, pois a ação coordenada entre braços,

\section{Método}

\section{Participantes}

Participaram do estudo, mediante assinatura do termo de consentimento, 21 atletas masculinos com idade entre 13 e 16 anos (14,52 $\pm 1,1$ anos), ou seja, das categorias infantil $(n=11)$ e juvenil ( $n$ $=10$ ), respectivamente, campeões e vice-campeões Paulista e Brasileiro de 2009. Para minimizar o efeito da preferência por algum padrão respiratório específico, foi utilizado como critério de inclusão da amostra ter preferência pelo padrão com inspiração unilateral para o lado direito ou esquerdo, identificado através da aplicação de questionário específico (Seifert, Chollet \& Allard, 2005). No entanto, vale destacar que nesta pesquisa somente um nadador declarou ser o lado esquerdo seu lado de inspiração preferido. E, em relação a este atleta, verificou-se que nas medidas analisadas os resultados não se diferenciaram daquele dos demais atletas. $\mathrm{O}$ presente estudo foi aprovado pelo Comitê de Ética e Pesquisa (CEP) da Escola de Educação Física e Esporte da Universidade de São Paulo (EEFE-USP). pernas, tronco e respiração deve ocorrer de forma simultânea e contínua. E, ao mesmo tempo, a execução de cada um destes componentes apresenta relativa autonomia frente ao nado como um todo (Freudenheim \& Madureira, 2006; Freudenheim et al., 2005; OliveIrA et al., 2009). Em suma, a partir de uma visão de sistemas hierárquicos multinivelares de subtodos semi-autônomos (hólons), que se ramificam em subtodos de uma ordem inferior (KoESTLER, 1969), a braçada, a princípio, pode ser considerada ela própria um 'todo' composto de duas partes (aérea e aquática). Expressam o todo, os aspectos que remetem à interação entre suas partes, ou seja, os aspectos invariantes da ação (padrão), e às suas partes, os aspectos que remetem aos componentes em si, ou seja, os aspectos variantes da ação (parametrização).

Neste sentido, o padrão da braçada de peritos, no que tange a sua organização temporal, deveria prevalecer sobre a modificação do padrão de respiração adotado. Portanto, diante do exposto, parece razoável supor que mediante uma modificação do padrão de respiração, não haja modificação no padrão espaçotemporal da braçada do nado "Crawl" (aspectos invariantes) de nadadores peritos, mas sim em aspectos relacionados à sua parametrização (aspectos variantes).

\section{Tarefa e delineamento}

A tarefa foi, saindo de dentro da piscina, nadar quatro tentativas de 25 m do nado "Crawl" em velocidade máxima em quatro condiçóes apresentadas em ordem randomizada e contrabalanceada. Para fins de análise, visando evitar efeito de saída e finalização, foram considerados os 10 metros centrais do percurso. As condiçōes foram: LP: Inspiração para lado preferido a cada ciclo de braçada; LNP: Inspiração para lado não preferido a cada ciclo de braçada; S: Sem inspiração - em apneia; e, B: Inspiração bilateral. Para evitar o efeito da fadiga e proporcionar tempo para preparação da próxima filmagem, foi fornecido intervalo de descanso entre tentativas de cinco minutos. A coleta ocorreu em dois dias, sendo que os 11 atletas da categoria infantil realizaram a tarefa no primeiro dia e os 10 atletas da categoria juvenil no segundo dia de coleta.

\section{Medidas}

Foram utilizadas como medidas que correspondem ao desempenho: tempo total $(\mathrm{Tt})$ - tempo em 
segundos (s) gasto para nadar a distância de $10 \mathrm{~m}$ em velocidade máxima; velocidade média $(\mathrm{Vm})$ velocidade em metros por segundo $(\mathrm{m} / \mathrm{s})$, consiste na razão entre a distância percorrida e o tempo gasto para percorrê-la $(\mathrm{Tt})$; frequência de braçadas $(\mathrm{Fb})$, baseada no ciclo de braçadas por minuto e calculada nos três ciclos completos de braçadas realizados no meio da piscina (Schnitzler; Seifert \& Chollet, 2011); comprimento de braçada (Cb) - em metros (m), consiste na razão entre a distância percorrida e o número de braçadas realizados nos $10 \mathrm{~m}$ centrais do percurso que reconhecidamente, é o método de uso mais comum utilizado para calcular o comprimento de braçada dos nadadores (MAgLisCHO, 2010).

O IdC foi utilizado como medida de coordenação das ações dos braços direito e esquerdo. Para o cálculo do IdC foi necessário analisar, conjuntamente, as fases aquática de entrada e pegada dos braços direito e esquerdo. Esta fase consiste da entrada do punho na água até o início do movimento da mão para trás. O início do movimento da mão para trás, identificado como o momento ("frame") em que a mão se encontrava abaixo da linha horizontal ao ombro, caracteriza o início do movimento propulsivo dos braços (Chollet, Chalies \& Chatard, 2000). O IdC foi utilizado como medida de interação das açóes dos braços direito e esquerdo e corresponde a média do IdCe e IdCd expressos em porcentagem de milissegundos (\%) da média do tempo total das braçadas direita e esquerda. O índice de coordenação esquerdo ( $\mathrm{IdCe}$ ) corresponde ao tempo entre o fim da propulsão do primeiro braço esquerdo e início da propulsão do primeiro braço direito. $O$ índice de coordenação direito (IdCd) corresponde ao tempo entre o fim da propulsão do primeiro braço direito e início da propulsão do segundo braço esquerdo.

Foram consideradas medidas dos aspectos invariantes da braçada, distribuição e variabilidade do "timing" relativo da fase aérea ( $T r$ Ae e Vtr Ae) e da fase aquática (Tr Aq e VTr Aq) dos braços direito e esquerdo. $\mathrm{O}$ ciclo da braçada de cada braço foi dividido em duas fases: aquática e aérea. $\mathrm{O}$ tempo total de movimento correspondeu à realização de um ciclo de braçada. A obtenção dos tempos foi feita mediante a contagem do número de quadros utilizando-se o "software" Kinovea 0.8.7. Com a duração total do ciclo e as durações de cada fase foi possível calcular o "timing" relativo das fases. O "timing" relativo consiste da porcentagem de milissegundos do tempo total de movimento (duração total da braçada) gasto em cada fase. Com a duração total do ciclo e as durações de cada fase da braçada foi possível calcular o timing relativo das fases aérea e aquática, bem como a variabilidade do "timing" relativo que é baseada no desvio padrão de três braçadas completas.

Por sua vez, foram utilizadas como medidas correspondentes aos aspectos variantes: tempo total em milissegundos (ms) e variabilidade do tempo total da fase aérea (Tt Ae e VTt Ae) e aquática (Tt Aq e VTt Aq) dos braços direito e esquerdo. Estas medidas tiveram como finalidade permitir a análise da consistência no padrão temporal das braçadas, bem como, a variabilidade desta consistência (SCHMIDT, 2003).

As medidas referentes ao IdC entre os braços e de organização temporal das braçadas foram calculadas pela média de três ciclos consecutivos de braçada (três ciclos por tentativa) em cada condição, nos $10 \mathrm{~m}$ centrais do percurso. Com esta determinação anulou-se o efeito que a saída para o nado e a sua finalização poderiam exercer sobre a organização temporal das braçadas.

\section{Instrumentos}

O experimento foi realizado em uma piscina de $25 \mathrm{~m}$ de comprimento por 12,5 de largura aberta e aquecida com profundidade de $5 \mathrm{~m}$.

Para possibilitar a filmagem por cima e por baixo da água foi utilizado um carrinho, especialmente construído para este fim. Este carrinho, com uma barra vertical de ferro galvanizado, foi colocado sobre um trilho de alumínio com $18 \mathrm{~m}$ de comprimento na lateral da piscina para acompanhar o nadador em velocidade máxima perpendicularmente nos 10 m centrais do percurso, com o mínimo de trepidação conforme ilustrado na FIGURA 1. Foi utilizada também uma raia de $25 \mathrm{~m}$, montada e instalada na piscina, com dois pontos brancos de referência para delimitar o espaço dos $10 \mathrm{~m}$ utilizados nas análises.

Para a captação das imagens aérea e aquática foram utilizadas duas filmadoras da marca Sony (60 fps), alinhadas verticalmente e sincronizadas através de um "flash" emitido por uma câmera fotográfica também da marca Sony (modelo DSC W50). A filmadora aérea, de modelo hdr xr 100 gravou a imagem que era enviada diretamente para o "notebook" da marca Toshiba através de uma placa de captura "easy" cap 2.0 que possibilitou a captura da imagem e o acompanhamento do nadador através do "software" Ulead VídeoStudio 10. Para a filmagem aquática, foi utilizada uma filmadora de modelo hdr sr11 que gravou a imagem aquática utilizada para a análise do IdC.

Para a fixação da filmadora aérea, foi utilizado um suporte de GPS na parte superior da barra vertical 
do carrinho para possibilitar ajustes no ângulo de filmagem. Já para a fixação da filmadora aquática foi necessária a construção de um olho de ferro galvanizado para diminuir a resistência com a água e assim facilitar o deslocamento do carro de filmagem no acompanhamento do nadador. Uma caixa estanque da marca Sony SPK-HCE foi utilizada para inserir a filmadora aquática e possibilitar a filmagem sem danos no equipamento.

Para sincronizar as imagens das filmadoras aérea e aquática, foi utilizado o "software" Ulead VídeoStudio 11. A partir das imagens geradas, para a obtenção dos tempos de desempenho e dos tempos em milissegundos de cada fase da braçada mediante a contagem do número de quadros, foi utilizado o "software" Kinovea 0.8.7. Para facilitar a identificação e análise dos tempos das fases das braçadas, foi utilizada uma fita velcro, para marcação do punho (cabeça da ulna).

Para facilitar a organização da coleta e a identificação dos nadadores nos registros, foram, respectivamente ao número de participantes, utilizadas 11 toucas de cor branca e amarela numeradas de um a 11 no primeiro dia e 10 toucas numeradas de um a 10 no segundo dia de coleta.

Para determinar a preferência do lado inspiratório e possibilitar a organização dos nadadores nas diferentes condiçôes experimentais foi aplicado o questionário para determinação do lado preferido adaptado de Seifert, Chollet e Allard (2005). Este questionário é composto por cinco questôes: 1) Qual lado e frequência respiratória você utiliza durante uma competição de 50 m?; 2) Qual o lado e frequência respiratória utilizada durante uma competição de 100 e 200 m?; 3) Qual o lado e frequência respiratória utilizada durante as competições de 400, 800 e 1500 m?; 4) Qual lado e frequência respiratória utilizada durante treinamento pesado?; e, 5) Qual o lado e frequência respiratória utilizada durante treinamento leve?

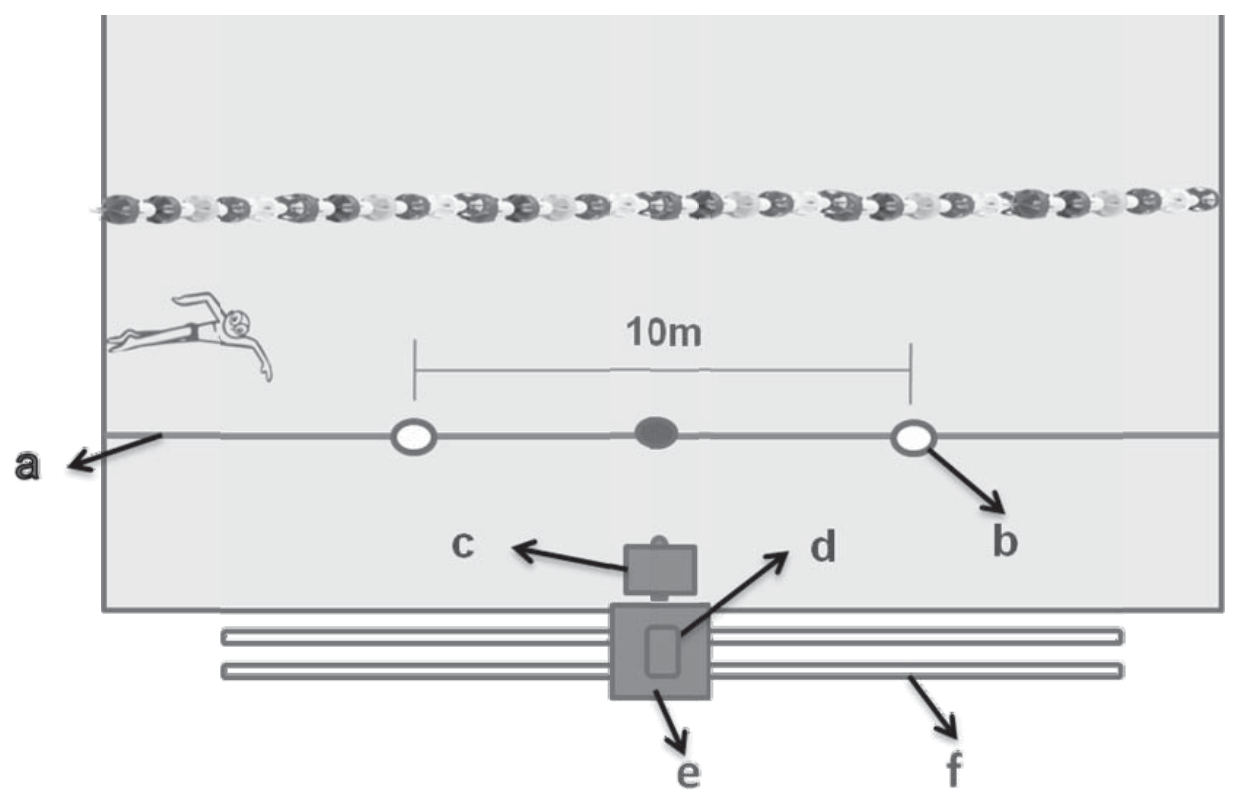

$a=$ raia de 25 metros; $b=$ ponto de referência dos 10 metros c = câmera e olho aquático; d = câmera aérea; e = carro de filmagem; $\mathrm{f}=$ trilho de alumínio de 18 metros.

FIGURA 1 - Ilustração dosinstrumentosutilizadosparaa análisedasfases aérea eaquática dabraçada donado "Crawl”.

\section{Tratamento de dados}

Considerando que todas as medidas utilizadas são de natureza intervalar e sendo 21 participantes analisados em quatro condiçôes, foram realizados testes de normalidade para verificar se os pressupostos da análise paramétrica seriam atendidos. Quando encontrada normalidade para todas as condições envolvidas $(\mathrm{p} \geq$ 0,05), foi realizada uma análise de variância (ANOVA "one-way") com medidas repetidas. Para localizar as diferenças apontadas pela ANOVA, foi aplicado o teste de "post hoc" de Bonferroni.

\section{Resultados}

Os participantes realizaram quatro execuções, uma tentativa em cada condição de respiração para a obtenção das medidas de desempenho. A seguir os resultados referentes às medidas de desempenho, de interação entre os braços e de organização temporal das braçadas. 


\section{Desempenho}

Conforme podemos observar na TABELA 1, a análise estatística detectou diferença significativa para o tempo total $(\mathrm{Tt})$ de deslocamento $\left[\mathrm{F}_{(3 ; 60)}\right.$ $=13,313 ; \mathrm{p}=0,001]$ entre a condição $S$ e as condiçōes LP, LNP e B, respectivamente, $p=0,022$; $\mathrm{p}=0,032$ e $\mathrm{p}=0,021 ;$ e, para a velocidade média $\left[\mathrm{F}_{(3 ; 60)}=12,601 ; \mathrm{p}=0,001\right]$ entre a condição $\mathrm{S}$ e as condiçoes $\mathrm{LP}, \mathrm{LNP}$ e $\mathrm{B}$, respectivamente, $\mathrm{p}=0,003$; $\mathrm{p}=0,001$ e $\mathrm{p}=0,005$.

Ainda em relação ao desempenho, a análise estatística detectou diferença significativa para a frequência de braçada $(\mathrm{Fb})\left[\mathrm{F}_{(3 ; 6)}=10,401 ; \mathrm{p}=0,001\right]$ e para $\mathrm{o}$ comprimento de braçada $(\mathrm{Cb})\left[\mathrm{F}_{(3 ; 6)}=9,729 ; \mathrm{p}=\right.$ $0,001]$ entre as condições $S$ e as condições LP, LNP e $B$, respectivamente, $p=0,007 ; p=0,001$ e $p=0,020$.

Desta forma, podemos verificar que na condição $\mathrm{S}$ a $\mathrm{Fb}$ tende a ser maior e o $\mathrm{Cb}$ menor em comparação com as condições LP, LNP e B (TABELA 1).

\section{Coordenação entre os braços direito e esquerdo}

Referente à coordenação entre os braços direito e esquerdo (IdC) (TABELA 1), a análise estatística não detectou diferença significativa entre as condições experimentais $\left[\mathrm{F}_{(3 ; 60)}=0,399 ; \mathrm{p}=0,774\right]$.

TABELA 1- Médiaedesvio padrão das medidas dedesempenho: Tempo total (Tt) em segundos(s); Velocidademédia (Vm) em metros por segundo (s) ; Frequência debraçadas (Fb); Comprimento debraçada (Cb) em metros (m); e, Índicedecoordenação entre os braços (IdC) em porcentagem demilissegundos (\%), nas condições de inspiração para o lado preferido (LP), lado não preferido (LNP), bilateral (B) e sem inspiração (S).

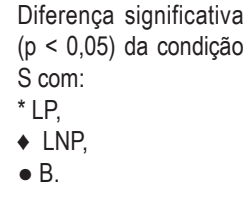

\begin{tabular}{cccccc}
\hline Condições & $\mathrm{Tt}(\mathrm{s})$ & $\mathrm{Vm}(\mathrm{m} / \mathrm{s})$ & $\mathrm{Fb}$ & $\mathrm{Cb}(\mathrm{m})$ & $\mathrm{IdC}(\%)$ \\
\hline $\mathrm{LP}$ & $6,06 \pm 0,32^{*}$ & $1,65 \pm 0,09 *$ & $51,49 \pm 2,29 *$ & $1,01 \pm 0,08 *$ & $1,68 \pm 2,15$ \\
$\mathrm{LNP}$ & $6,14 \pm 0,36 \bullet$ & $1,63 \pm 0,10 \bullet$ & $50,74 \pm 2,26 \bullet$ & $1,02 \pm 0,07 \bullet$ & $2,80 \pm 3,13$ \\
$\mathrm{~B}$ & $6,05 \pm 0,33 \bullet$ & $1,66 \pm 0,09 \bullet$ & $52,16 \pm 2,34 \bullet$ & $0,99 \pm 0,07 \bullet$ & $2,56 \pm 2,60$ \\
$\mathrm{~S}$ & $5,95 \pm 0,38$ & $1,68 \pm 0,09$ & $53,75 \pm 2,40$ & $1,00 \pm 0,00$ & $2,09 \pm 2,90$ \\
\hline
\end{tabular}

\section{Organização temporal das braçadas}

\section{Aspectos invariantes}

Para as variáveis relacionadas aos aspectos invariantes (TABELA 2), também não foram detectadas diferenças significativas entre as condições experimentais, respectivamente, $\left[\mathrm{F}_{(3 ; 60)}=0,636 ; \mathrm{p}=0,595\right]$ $\mathrm{e}\left[\mathrm{F}_{(3 ; 60)}=0,314 ; \mathrm{p}=0,815\right]$ para o "timing" relativo aéreo e, $\left[\mathrm{F}_{(3 ; 60)}=0,636 ; \mathrm{p}=0,595\right]$ e $[\mathrm{F}(3: 60)=0,314$; $\mathrm{p}=0,815]$ para o "timing" relativo aquático, dos braços direito e esquerdo.

Com relação à variabilidade do timing relativo aéreo (VTr aéreo) e aquático (VTr aquático), não houve diferença significativa entre as condiçōes experimentais, respectivamente, para os braços direito e esquerdo aéreo $\left[\mathrm{F}_{(3 ; 60)}=0,608 \mathrm{p}=0,613\right]$ e $\left[\mathrm{F}_{(3 ; 60)}=0,625 ; \mathrm{p}=0,602\right]$ e para os braços direito e esquerdo aquático $\left[\mathrm{F}_{(3 ; 60)}=\right.$ $0,608 ; \mathrm{p}=0,613]$ e $\left[\mathrm{F}_{(3 ; 60)}=0,625 ; \mathrm{p}=0,602\right]$.

\section{Aspectos variantes}

Conforme apresentado na TABELA 3, em relação ao tempo total aéreo ( $\mathrm{Tt}$ aéreo) dos braços direito e esquerdo dos nadadores, foi detectada diferença significativa entre as condições experimentais $\left[\mathrm{F}_{(3 ; 60)}\right.$
$=3,867 ; \mathrm{p}=0,014]$ entre a condição $S$ e as condições LNP e LP para o braço direito (respectivamente, $\mathrm{p}$ $=0,028$ e $\mathrm{p}=0,001)$ e para o braço esquerdo, entre as condições $S$ e LNP $(p=0,023)$.

Em relação à variabilidade do tempo total aéreo (VTt aéreo), não foram detectadas diferenças significativas entre as condições experimentais para o braço direito $\left[\mathrm{F}_{(3 ; 54)}=1,586 ; \mathrm{p}=0,302\right]$ e para o esquerdo $\left[\mathrm{F}_{(3 ; 57)}=0,623 ; \mathrm{p}=0,603\right]$.

Para o tempo total aquático ( $\mathrm{Tt}$ aquático) e para a variabilidade do tempo total aquático (VTt aquático) dos braços direito e esquerdo, também apresentados na TABELA 3, a análise inferencial não detectou diferença significativa entre as condições experimentais, respectivamente, para o braço direito $\left[\mathrm{F}_{(3 ; 60)}=2,710 ; \mathrm{p}=0,053\right]$ e $\left[\mathrm{F}_{(3 ; 51)}=2,586 ; \mathrm{p}=\right.$ $0,063]$ e para o braço esquerdo $\left[\mathrm{F}_{(3 ; 60)}=2,449 ; \mathrm{p}=\right.$ $0,072]$ e $\left[\mathrm{F}_{(3 ; 51)}=1,313 ; \mathrm{p}=0,280\right]$.

Já para o tempo total das braçadas ( $\mathrm{Tt}$ braçada) direito e esquerdo, a análise estatística detectou diferença significativa para o braço direito $\left[\mathrm{F}_{(3 ; 60)}=\right.$ $8,144 ; \mathrm{p}=0,000]$ e braço esquerdo $\left[\mathrm{F}_{(3 ; 60)}=9,243\right.$; $p=0,000]$, especificamente, entre a condição LNP e B e entre as condiçōes $S$ e LNP para os braços direito $(\mathrm{p}=0,020, \mathrm{p}=0,004)$ e braço esquerdo $(\mathrm{p}$ $=0,016, \mathrm{p}=0,002)$. 
Por fim, verifica-se também na TABELA 3, que para a variabilidade do tempo total da braçada (VTt braçada) direita, foi detectada diferença significativa $\left[\mathrm{F}_{(3 ; 60)}=5,724 ; \mathrm{p}=0,002\right]$ entre a condição $\mathrm{S}$ e as condiçôes LP e LNP, respectivamente, $\mathrm{p}=0,002 \mathrm{e} \mathrm{p}$ $=0,030$. Para o braço esquerdo a análise inferencial não detectou diferença significativa $\left[\mathrm{F}_{(3 ; 45)}=1,081\right.$; $\mathrm{p}=0,360]$.

TABELA 2 - Média e desvio padrão da porcentagem dos milissegundos (\%ms) referenteaos aspectos invariantes: “Timing" relativo aéreo (Tr aéreo); Variabilidade do "Timing" relativo aéreo (VTr aéreo); "Timing" relativo aquático (Tr aquático); Variabilidade do "Timing" relativo aquático (VTr aquático) para o braço direito e esquerdo nas condições de inspiração para o lado preferido (LP), lado não preferido (LNP), bilateral (B) e sem inspiração (S).

\begin{tabular}{|c|c|c|c|c|}
\hline \multicolumn{5}{|c|}{ BRAÇO DIREITO } \\
\hline Condiçóes & $\begin{array}{c}\text { Tr aéreo } \\
(\%)\end{array}$ & $\begin{array}{c}\text { VTr aéreo } \\
(\%)\end{array}$ & $\begin{array}{c}\text { Tr aquático } \\
(\%)\end{array}$ & $\begin{array}{c}\text { VTr aquático } \\
(\%)\end{array}$ \\
\hline LP & $33,49 \pm 3,07$ & $2,06 \pm 1,15$ & $66,51 \pm 3,07$ & $2,06 \pm 1,15$ \\
\hline LNP & $32,80 \pm 3,13$ & $2,31 \pm 1,12$ & $67,20 \pm 3,13$ & $2,31 \pm 1,12$ \\
\hline $\mathrm{B}$ & $33,14 \pm 4,40$ & $2,68 \pm 1,77$ & $66,86 \pm 4,40$ & $2,68 \pm 1,77$ \\
\hline$S$ & $32,39 \pm 3,32$ & $2,50 \pm 1,13$ & $67,61 \pm 3,32$ & $2,50 \pm 1,13$ \\
\hline \multicolumn{5}{|c|}{ BRAÇO ESQUERDO } \\
\hline Condições & $\begin{array}{c}\text { Tr aéreo } \\
(\%)\end{array}$ & $\begin{array}{c}\text { VTr aéreo } \\
(\%)\end{array}$ & $\begin{array}{c}\text { Tr aquático } \\
(\%)\end{array}$ & $\begin{array}{c}\text { VTr aquático } \\
(\%)\end{array}$ \\
\hline LP & $33,47 \pm 4,02$ & $2,22 \pm 1,04$ & $66,53 \pm 4,02$ & $2,22 \pm 1,04$ \\
\hline LNP & $34,06 \pm 4,04$ & $1,88 \pm 0,77$ & $65,94 \pm 4,04$ & $1,88 \pm 0,77$ \\
\hline $\mathrm{B}$ & $33,61 \pm 3,05$ & $1,91 \pm 1,18$ & $66,39 \pm 3,05$ & $1,91 \pm 1,18$ \\
\hline$S$ & $33,24 \pm 2,88$ & $2,15 \pm 1,15$ & $66,76 \pm 2,88$ & $2,15 \pm 1,15$ \\
\hline
\end{tabular}

TABELA 3 - Média e desvio padrão dos milissegundos (ms) referente aos aspectos variantes: Tempo total aéreo (Tt aéreo); Variabilidade do Tempo total aéreo (VTt aéreo); Tempo total aquático (Tt aquático); Variabilidade do Tempo total aquático (VTt aquático); Tempo total das braçadas (Tt braçada); Variabilidade do Tempo total da braçada (VTt braçada) para o braço direito e esquerdo nas condições deinspiração para o lado preferido (LP), lado não preferido (LNP), bilateral (B) esem inspiração (S).

\begin{tabular}{lcccccc}
\hline \multicolumn{7}{c}{ BRAÇO DIREITO } \\
\hline Condiçóes & $\begin{array}{c}\text { Tt aéreo } \\
(\mathbf{m s})\end{array}$ & $\begin{array}{c}\text { VTt aéreo } \\
(\mathbf{m s})\end{array}$ & $\begin{array}{c}\text { Tt aquático } \\
(\mathbf{m})\end{array}$ & $\begin{array}{c}\text { VTt aquático } \\
(\mathbf{m s})\end{array}$ & $\begin{array}{c}\text { Tt braçada } \\
(\mathbf{m s})\end{array}$ & $\begin{array}{c}\text { VTt braçada } \\
(\mathbf{m})\end{array}$ \\
\hline LP & $156,66 \pm 14,68^{*}$ & $10,02 \pm 3,92$ & $311,9 \pm 28,08$ & $10,73 \pm 3,18$ & $468,57 \pm 29,45$ & $13,11 \pm 5,74 *$ \\
LNP & $156,66 \pm 15,06 \star$ & $11,08 \pm 6,00$ & $322,22 \pm 32,36$ & $12,4 \pm 4,71$ & $478,89 \pm 34,11 \star$ & $13,23 \pm 6,81$ \\
B & $152,70 \pm 19,20$ & $13,15 \pm 7,87$ & $308,73 \pm 31,01$ & $14,05 \pm 5,16$ & $461,43 \pm 27,64 \boldsymbol{1}$ & $14,97 \pm 8,44$ \\
S & $145,87 \pm 16,22$ & $13,36 \pm 5,57$ & $304,92 \pm 27,30$ & $10,78 \pm 4,31$ & $450,79 \pm 29,59$ & $20,36 \pm 8,54$ \\
\hline
\end{tabular}

Diferença significativa $(p<0,05)$ da condição $S$ com: ${ }^{*} \mathrm{LP}$,

$\checkmark$ LNP, - $\mathrm{B}$,

$\Delta$ entre a condição LNP e B. $(p<0,05)$ da condição $S$ com: * LP, - LNP, B.

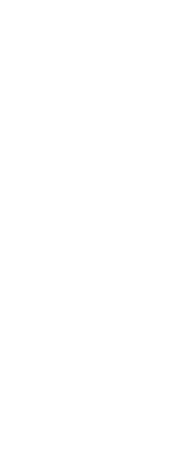

\begin{tabular}{lcccccc}
\hline \multicolumn{7}{c}{ BRAÇO ESQUERDO } \\
Condiçóes & $\begin{array}{c}\text { Tt aéreo } \\
(\mathbf{m})\end{array}$ & $\begin{array}{c}\text { VTt aéreo } \\
(\mathbf{m})\end{array}$ & $\begin{array}{c}\text { Tt aquático } \\
(\mathbf{m})\end{array}$ & $\begin{array}{c}\text { VTt aquático } \\
(\mathbf{m})\end{array}$ & $\begin{array}{c}\text { Tt braçada } \\
(\mathbf{m s})\end{array}$ & $\begin{array}{c}\text { VTt braçada } \\
(\mathbf{m s})\end{array}$ \\
\hline LP & $155,56 \pm 19,25$ & $11,87 \pm 5,51$ & $309,84 \pm 29,54$ & $10,52 \pm 4,84$ & $465,40 \pm 28,00$ & $13,79 \pm 6,98$ \\
LNP & $162,38 \pm 19,27 \bullet$ & $9,99 \pm 5,18$ & $315,4 \pm 32,51$ & $10,87 \pm 4,29$ & $477,78 \pm 32,73 \bullet$ & $11,99 \pm 6,18$ \\
B & $154,97 \pm 16,71$ & $13,11 \pm 9,00$ & $304,87 \pm 22,00$ & $12,81 \pm 7,95$ & $459,84 \pm 27,62 \bullet$ & $16,94 \pm 11,24$ \\
S & $148,89 \pm 14,96$ & $10,49 \pm 8,92$ & $299,21 \pm 25,36$ & $12,34 \pm 3,90$ & $448,10 \pm 29,95$ & $14,64 \pm 06,64$ \\
\hline
\end{tabular}




\section{Discussão}

O objetivo do presente estudo foi investigar se o desempenho e a organização temporal das braçadas do nado "Crawl" são afetados pelos padrões respiratórios: sem inspiração (apneia), com inspiração unilateral (LP e LNP) e bilateral. A amostra compreendeu atletas masculinos das categorias infantil e juvenil, todos com preferência pelo padrão respiratório unilateral.

Para a análise foram consideradas medidas que correspondem ao desempenho (tempo gasto para percorrer a distância de $10 \mathrm{~m}$ em velocidade máxima, velocidade média, $\mathrm{Fb}$ e $\mathrm{Cb}$ ); aos aspectos invariantes ("timing" relativo das fases aérea e aquática dos braços direito e esquerdo), aos variantes (tempo total da fase aérea e aquática do braço direito e esquerdo, tempo total do braço direito e esquerdo), e à interação entre os braços (IdC) do nado "Crawl".

Os resultados mostraram que o padrão respiratório afetou o desempenho dos atletas jovens. Eles precisaram de menos tempo e, portanto, foram também mais velozes para percorrer $10 \mathrm{~m}$ na condição $S$ (sem inspiração) que nas demais condições. Dessa forma, mesmo no caso de nadadores peritos jovens, podemos observar que efetuar a inspiração, independente do padrão adotado, afeta velocidade e tempo de nado. Assim, em termos desempenho, no que diz respeito à velocidade e tempo para percorrer $10 \mathrm{~m}$ em velocidade máxima, em comparação com qualquer das condiçôes com inspiração a condição de nadar "Crawl" em apneia é mais vantajosa.

Importante ressaltar que não há na literatura estudos que tenham apresentado dados em relação aos efeitos de padrões de respiração no tempo e velocidade de nadadores. $\mathrm{Na}$ maior parte dos estudos até então desenvolvidos, a velocidade foi variável independente, pois os atletas foram solicitados a nadar simulando velocidade empregada em determinadas provas (LeRDA, Cardelli \& CHOllet, 2001; PAYTON et al., 1999; Vezos et al., 2007). No único estudo em que a velocidade foi variável dependente (SEIFERT et al., 2008), não foi verificada diferença na velocidade dos nadadores em função das condições sem e com inspiração. Mas, neste estudo o nado "Crawl" foi descaracterizado, pois os nadadores nadaram com as pernas atadas. $\mathrm{O}$ presente estudo se diferencia dos demais pois investigou o efeito do padrão de respiração no desempenho do nado "Crawl”, em situação mais próxima do real, ou seja, em velocidade máxima real, sem descaracterizar o nado. Talvez por isso, os resultados do presente estudo não corroborem com os encontrados por SEIFERT et al. (2008).
Os resultados encontrados, que em termos de velocidade e tempo, é mais vantajosa a condição de nadar em apneia comparada as demais condiçôes, podem ser explicados pelo arrasto ativo causado pela movimentação do corpo em função da ação respiratória, e consequente aumento significativo no tempo de deslocamento dos nadadores. Fortalece este argumento o fato de que nadar em apneia resulta numa menor oscilação do tronco (MAGLISCHO, 1999, 2003).

Ainda a respeito das variáveis de desempenho, verificou-se que na condição $S$ apresentou maior $\mathrm{Fb}$ e menor $\mathrm{Cb}$ comparado aos padrōes de inspiração LP, LNP e B. Assim, o conjunto de resultados aponta que em relação à eficiência da braçada, mensurada pela frequência e comprimento de braçada, a condição $S$ é a mais eficiente comparada às condições de inspiração e LP, LNP e B.

Portanto, os resultados deste estudo indicam que o padrão preferido de respiração, não necessariamente, corresponde ao mais eficiente em termos do desempenho, e que o padrão $S$ pode servir como estratégia tática para nadadores velocistas.

Este estudo também teve como objetivo investigar os efeitos de diferentes padrões respiratórios na organização temporal das braçadas do nado "Crawl". Nesse sentido, foram consideradas medidas correspondentes à consistência do padrão temporal das braçadas direita e esquerda (Coordenação entre braços e aspectos invariantes) bem como à variabilidade (aspectos variantes) da organização temporal dos braços direito e esquerdo.

Os resultados mostraram que o padrão respiratório não afetou a consistência da organização temporal das braçadas, mas que afetou sua variabilidade, ou seja, as medidas correspondentes aos aspectos variantes.

No que diz respeito à consistência, na medida de $\mathrm{IdC}$, os resultados mostraram que não houve diferença significativa entre as condições experimentais. Portanto, os resultados do presente estudo indicam que, independente do padrão respiratório utilizado, atletas jovens mantêm o padrão de coordenação entre os braços. Esses resultados não corroboram com os de Lerda, Cardelli e Chollet (2001) nos quais os atletas apresentaram IdC maior na condição em apneia comparado ao da condição com inspiração a cada ciclo de braçada. Esse resultado levou os autores a concluírem que inspirar enquanto se nada aumenta a descontinuidade das ações propulsivas dos braços. Esta diferença nos resultados pode ser 
explicada pelo fato de os nadadores no estudo de Lerda, Cardelli e Chollet (2001) serem mais velhos e experientes que os do presente estudo e, por isso, provavelmente, capazes de tirar vantagem da condição aumentando a continuidade (sobreposição) da ação dos braços quando em apneia.

No presente estudo, também nas medidas que correspondem aos aspectos invariantes da braçada do nado "Crawl" (consistência), "timing" relativo das fases aérea e aquática dos braços direito e esquerdo, bem como sua variabilidade, não houve efeito das condições experimentais.

Dessa forma pode-se considerar que, diante da modificação do padrão respiratório, nadadores peritos jovens mantêm a estrutura da organização temporal do movimento (padrão) das braçadas inalterada. Este resultado vem ao encontro de uma característica organizacional dos sistemas hierárquicos abertos: a diferenciação, expressa por uma relativa autonomia das partes integrantes do sistema frente às demais, embora não suficiente, é condição necessária para que haja interação (BERTALANFFY, 1977). Portanto interação e autonomia são atributos complementares.

Esta característica também foi verificada em relação a diferentes ritmos de pernada (APOLINÁrio, Oliveira, Marques, Corrêa e Freudenheim (2011). Nesse estudo, os autores investigaram os efeitos do ritmo da pernada na organização temporal da braçada do nado "Crawl". Participaram 12 adultos, entre 21 e 51 anos de idade. A tarefa consistiu em percorrer 25 metros nadando "Crawl" em velocidade lenta, em três ritmos de pernada (duas, quatro e seis pernadas por ciclo de braços). Como não foram encontradas diferenças significativas na organização temporal da braçada do nado "Crawl" em função do ritmo de pernada, concluiu-se que o ritmo da pernada não interfere na organização temporal da braçada.

Com base nesses resultados, podemos observar que os nadadores apresentam organização temporal das braçadas do nado "Crawl" similares e estáveis, ou seja, mantêm os aspectos invariantes frente à modificação do padrão respiratório. Esse resultado mostra que, em se tratando de atletas jovens, uma modificação na estrutura de um componente da tarefa (padrão de respiração) pode não gerar alteração na estrutura de outro componente (padrão temporal das braçadas). No entanto, em futuros estudos, dever-se-á considerar o efeito de diferentes padrôes de respiração e de pernada no desempenho e na braçada do nado "Crawl".
Por sua vez, nas medidas correspondentes aos aspectos variantes, foram encontradas diferenças nas medidas de tempo total da fase aérea, entre a condição $S$ e as condiçôes LNP e LP para o braço direito, e entre as condiçôes S e LNP, para o braço esquerdo. Dessa forma, podemos afirmar que, a ação respiratória afeta a fase aérea da braçada dos nadadores jovens, mas não a fase aquática, nem a variabilidade de ambas.

Considerando os resultados em conjunto, verifica-se que o melhor desempenho na condição $S$, quando comparado às condições LP e LNP, pode ser atribuído à diferença no tempo total da fase aérea da braçada, ou seja, na fase de recuperação. Esta sensibilidade da fase aérea com modificações do padrão respiratório corrobora as observadas nos estudos realizados com modificação paramétrica na velocidade de nado de crianças MADUREIRA (2006) e atletas SiLva (2008). Nestes estudos os ajustes frente às modificaçôes da velocidade de nado, também foram efetuados mediante modificaçôes nos aspectos variantes, relacionados à fase aérea. Nesse sentido, considerando estes estudos em conjunto pode-se concluir que diferenças no desempenho (velocidade) ocorrem mediante ajustes dos aspectos variantes, mais especificamente, relacionados à fase aérea da braçada. Portanto, que atletas de elite parecem efetuar os ajustes considerando economia de recursos.

Ainda em relação à fase aérea dos braços direito e esquerdo, observamos que, somente a condição B não se diferenciou das demais. A maior eficiência da braçada (frequência e comprimento) nesta condição de inspiração sobre as demais (LP e LNP), pode ser explicada pelo fato de os nadadores terem efetuado a ação dos braços com um tempo total da fase aérea, mais semelhante ao da condição $S$.

Em relação ao tempo total das braçadas, os resultados mostram que houve aumento em ambos os braços da condição $S$ para a LNP e que para o braço direito, além desta diferença, houve aumento também de B para LNP. Estes resultados permitem afirmar que a ação respiratória unilateral para o lado não preferido (LNP) causa aumento do tempo total da ação dos braços (direito e esquerdo) dos nadadores e, consequentemente, prejudica o desempenho dos mesmos nesta condição. A condição LP, nesta variável, não se diferenciou das demais. Este é mais um indicativo de vantagem da adoção da condição de inspiração B, sobre as demais, inclusive daquela (LP), declarada de preferência pelos nadadores.

Em síntese, os resultados referentes às medidas que correspondem à parametrização, em conjunto com as que remetem à estrutura do padrão da braçada, 
permitem inferir que as alterações de desempenho entre as condições ocorreram mediante ajustes nos aspectos variantes da braçada do nado "Crawl".

Com base nos resultados obtidos e considerando as delimitações da presente pesquisa, pode-se concluir que: a) $\mathrm{O}$ padrão respiratório afeta o desempenho dos atletas jovens. Em termos de velocidade, tempo, frequência e comprimento de braçada, é mais vantajosa a condição de nadar "Crawl" em apneia que em qualquer das condições com inspiração. Portanto, o padrão preferido de respiração, não necessariamente, corresponde ao mais eficiente em termos do desempenho; b) Diante da modificação do padrão respiratório, nadadores peritos jovens mantêm a estrutura temporal do movimento (padrão) das braçadas direita e esquerda (aspectos invariantes da braçada) bem como a interação entre os braços inalterada enquanto efetuam ajustes na fase aérea da braçada (aspectos variantes). Assim sendo, atletas jovens de elite apresentam braçada com relativa autonomia frente ao componente respiração e nadam efetuando ajustes considerando a economia de recursos.

Estes resultados sugerem que professores e técnicos de Natação deveriam incentivar a prática do padrão sem respiração, pois em termos de eficiência é o que apresentou melhor desempenho comparado aos padrões com ação respiratória, o que pode se tornar decisivo para os atletas de alto nível em provas de curta distância. Estes resultados remetem à necessidade de, em estudos futuros, investigar os efeitos de diferentes frequências respiratórias no desempenho e na organização temporal das braçadas do nado "Crawl" de atletas jovens de elite.

\begin{abstract}
Effects of different breathing patterns on the performance and the arm stroke in the front Crawl

The aim of this study was to investigate the effects of different breathing patterns on the performance and the arm stroke in the front Crawl of elite athletes. Twenty-one young male athletes were recruited to the study. They were filmed swimming 25 meters at maximum speed using the front Crawl stroke, under four breathing conditions: preferential inhalation side (PS); nonpreferential inhalation side (NPS); bilateral inhalation (B) and without inhalation (N). Two digital video cameras (60fps) captured the images that were analyzed using the software Kinovea 0.8 .7 in the central 10 meters of the length. Performance and temporal organization of stroke measures were considered for analysis. The results showed that the breathing pattern affects the performance of young elite athletes. In terms of speed, time, stroke rate and stroke length, comparing to any of the inhalation conditions (B, PS and NPS), to swim front Crawl stroke in apnea $(\mathrm{N})$ is more advantageous. Therefore, the preferred breathing pattern does not necessarily correspond to the most efficient one in terms of performance. Thus, swimming teachers and coaches should encourage the practice and the usage of the apnea pattern as a tactical strategy for swimmers sprinters. Regarding arm stroke, the results showed that, given the change in the breathing pattern, young expert swimmers maintain the temporal structure of the right and left arm strokes (invariant stroke features) as well as the coordination between the arms unchanged while adjusting the aerial phase of the stroke (variant stroke features). Thus, young elite athletes exhibit a stroke with relative autonomy regarding the breath component and swim making adjustments considering the saving of resources.
\end{abstract}

UnITERMS: Swimming; Front Crawl stroke; Motor behavior.

\title{
Referências
}

APOLINÁRIO, M.R.; OLIVEIRA, T.A.C.; MARQUES, M.T.; CORRÊA, U.C.; FREUDENHEIM, A.M. Efeito da modificação dos ritmos de pernada na braçada do nado crawl. Revista Mackenzie de Educação Física e Esporte, São Paulo, v.10, n.1, p.11-8, 2011. APOLINÁRIO, M.R.; SILVA, C.G.S.; TERTULIANO, I.W.; OLIVEIRA, T.A.C.; MARQUES, M.T.; FREUDENHEIM, A.M. Efeito da modificação estrutural na braçada do nado crawl: um estudo exploratório. In: SIMPÓSIO INTERNACIONAL DE CIÊNCIAS DO ESPORTE, 30., 2007, São Paulo. Anais... São Paulo: 2007. p.248. 
BARTLETT, F.C. Remembering: a study in experimental and social psychology. Cambridge: Cambridge University Press, 1932. BERTALANFFY L.V. Teoria geral dos sistemas. Petrópolis: Vozes, 1977.

CHOLLET, D.; CHALIES, S.; CHATARD, J.C. A new index of coordination for the crawl: description and usefulness. International Journal of Sports Medicine, Stuttgart, v.21, p.54-9, 2000.

DESCHODT, V.J.; ARSAC, L.M.; ROUARD, A.H. Relative contribution of arms and legs in humans to propulsion in $25 \mathrm{~m}$ sprint front-crawl swimming. European Journal of Applied Physiology,Berlin, v.80, n.3, p.192-9, 1999.

FREUDENHEIM, A.M.; BASSO, L.; XAVIER FILHO, E; SILVA, C.G; MADUREIRA, F.; MANOEL, E.J. Organização temporal da braçada do nado crawl: iniciantes "versus" avançados. Revista Brasileira de Ciência e Movimento, São Caetano do Sul, v.15, n.2, p.75-84, 2005.

FREUDENHEIM, A.M.; MADUREIRA, F. Natação: análise e ensino do nado crawl. In: TANI, G.; BENTO, J.O; SOUZA, P.R.D. (Orgs.). Pedagogia do desporto. Rio de Janeiro: Guanabara Koogan, 2006. p.372-83.

HEUER, H. Invariant relative timing in motor program theory. In: FAGARD, J.; WOLF, P.H. (Eds.). The development of timing control and temporal organization in coordinated action: invariant relative timing, rhythms and coordination. Amsterdam: North-Holland, 1991. p.37-68.

KOESTLER, A. O fantasma da máquina. Rio de Janeiro: Zahar, 1969.

LERDA, R.; CARDELLI, C.; CHOLLET, D. Analysis of interaction between breathing and arm actions in front crawl. Journal of Human Movement Studies, London, v.40, p.129-44, 2001.

MADUREIRA, F. B. Efeito da modificaçáo da tarefa na braçada do nado crawl em indivíduos com níveis de habilidades distintos. 2006. 76f. Dissertação (Mestrado) - Escola de Educação Física e Esporte, Universidade de São Paulo, São Paulo, 2006. MAGLISCHO, E. W. Nadando ainda mais rápido. São Paulo: Manole, 1999.

Nadando o mais rápido possível. São Paulo: Manole, 2010.

Swimming fastest. Champaign: Human Kinetics, 2003.

OLIVEIRA, T.A.C.; APOLINÁRIO, M.R.; FREUDENHEIM, A.M.; CORREA, U.C. Análise sistêmica do nado crawl. Brasilian Journal of Motor Behavior, São Paulo, v.4, n.1, p.15-21, 2009.

PAYTON, C.J.; BARTLETT, R.M.; BALTZOPOULOS, V.; COOMBS, R. Upper extremity kinematics and body roll during preferred side breathing and breath-holding front crawl swimming. Journal of Sports Sciences, London, v.17, p.689-96, 1999. SCHMIDT, R.A. Motor schema theory after 27 years: reflections and implications for a new theory. Research Quarterly for Exercise and Sport, Washington, v.74, n.4, p.366-75, 2003.

The search for invariance in skilled movement behavior. Research Quarterly for Exercise and Sport, Washington, v. 56, n. 2, p.188-200, 1985.

SCHNITZLER, C.; SEIFERT, L.; CHOLLET, D. Arm coordination and performance level in the 400m front crawl. Research Quarterly for Exercise and Sport, Washington, v.82, n.1, p.1-8, 2011.

SEIFERT, L.; CHEHENSSE, A.; CHOLLET, C.T.; LEMAITRE, F.; CHOLLET, D. Effect of breathing pattern on arm coordination symmetry in front crawl. Journal of Strength and Conditioning Research, Champaign, v.22, p.1670-6, 2008. SEIFERT, L.; CHOLLET, D.; ALLARD, P. Arm coordination symmetry and breathing effect in front crawl. Human Movement Science, v.24, p.234-56, 2005.

SILVA, C.G.S. Consistência e variabilidade do nado crawl em indivíduos habilidosos. 2008. 75f. Dissertação (Mestrado) - Escola de Educação Física e Esporte, Universidade de São Paulo, São Paulo, 2008.

VEZOS, N.; GOURGOULIS, V.; AGGELOUSSIS, N.; KASIMATIS, P.; CHRISTOFORIDIS, C.; MAVROMATIS, G. Underwater stroke kinematics during breathing and breath-holding front crawl swimming. Journal Sports Science and Medicine, Bursa, v.6, p.58-62, 2007.

ENDEREÇO

Marcos Roberto Apolinário

Escola de Educação Física e Esporte - USP

Av. Prof. Mello de Moraes, 65

05508-030 - São Paulo - SP - BRASIL e-mail: apolinario@usp.br

Recebido para publicação: 14/ 06/ 2011

1a. Revisão: 26/ 09/2011

2a. Revisão: 04/11/ 2011

Aceito: 06/01/2012 